\title{
INFLUÊNCIA DO AMBIENTE DOMICILIAR NA OCORRÊNCIA DE INFECÇÕES RESPIRATÓRIAS AGUDAS EM CRIANÇAS MENORES DE 5 ANOS DE IDADE NO SUL DE MOÇAMBIQUE
}

\section{ARTIGO ORIGINAL}

SAMUEL, Wilson ${ }^{1}$, LANGA, Sílvia 2 ${ }^{2}$, SCARLET, Maria Perpetua Janeiro ${ }^{3}$, MONJANE, Nércia Eugénio ${ }^{4}$, MONTEIRO, Leonel Govindo ${ }^{5}$

SAMUEL, Wilson. Et al. Influência do ambiente domiciliar na ocorrência de infecções respiratórias agudas em crianças menores de $\mathbf{5}$ anos de idade no Sul de Moçambique. Revista Científica Multidisciplinar Núcleo do Conhecimento. Ano 06, Ed. 05, Vol. 03, pp. 73-88. Maio de 2021. ISSN: 2448-0959, Link de acesso: https://www.nucleodoconhecimento.com.br/saude/ambiente-domiciliar,

DOI: 10.32749/nucleodoconhecimento.com.br/saude/ambiente-domiciliar

\section{RESUMO}

As infecções respiratórias agudas estão entre as principais causas de morbimortalidade em crianças menores de 5 anos de idade em Moçambique. $O$ risco de mortalidade infantil nesses pais é devido á exposição a fatores ambientais. Este estudo tem como objetivo estabelecer a proporção de infecção respiratória aguda e

\footnotetext{
${ }^{1}$ Graduado em Biologia em Saúde e pós- graduação.

${ }^{2}$ Doutora em Ciências Biológicas.

${ }^{3}$ Doutora em Ecologia.

${ }^{4}$ Graduada em Medicina.

${ }^{5}$ Orientador. Mestre em Epidemiologia do Campo e Laboratorial.
}

RC: 84483

Disponível em: https://www.nucleodoconhecimento.com.br/saude/ambiente-domiciliar 
os fatores de risco relacionados às condições do ambiente domiciliar em crianças de 0 a 59 meses na Localidade de Beleluane, Província de Maputo, no sul de Moçambique. Para tal, entre Maio e Novembro de 2016, foi conduzido um estudo transversal em 330 agregados familiares com crianças menores de 5 anos de idade. Foram aplicados questionários padronizados aos chefes de agregados familiares daquela localidade, e coletaram-se informações sobre características ambientais domiciliares, condições de habitação e nível socioeconómico da família e, adicionalmente, foram recolhidos dados sobre as principais queixas durante a assistência médica de crianças menores de 5 anos no Centro de Saúde local. Para análise dos principais fatores de riscos foi realizada regressão logística. Foi observado que 45.9 a $68.2 \%$ das crianças procuram cuidados de saúde devido a infecção respiratória aguda, representando a primeira razão de busca dos cuidados de saúde. A nível dos agregados familiares a infecção respiratória aguda foi referida em $41.5 \%$. Os principais fatores de risco identificados foram: quatro ou mais pessoas dividindo o quarto da criança $(\mathrm{OR}=4.56 ; 95 \% \mathrm{Cl}: 2.49-8.35)$, animais no interior do domicílio (OR = 6.64; 95\%Cl: $3.11-14.16)$, uso de combustíveis sólidos $(\mathrm{OR}=4.22 ; 95 \% \mathrm{Cl}$ : $2.02-8.80)$, tabagismo domiciliar $(\mathrm{OR}=5.97 ; 95 \% \mathrm{Cl}: 3.21-11.09)$, e renda do agregado familiar mensal menor do que um salário mínimo ( $\$ 58,00)(O R=10.265$; $95 \%$ Cl: 5.168 - 20.391). Os resultados deste estudo mostram que as características ambientais domiciliares e a renda familiar estão entre os principais determinantes das infecções respiratórias agudas nesse grupo etário no sul de Moçambique.

Palavras-chave: Ambiente domiciliar, Infecção respiratória aguda, Fatores de riscos.

\section{INTRODUÇÃO}

As infecções respiratórias agudas (IRAs) estão entre as principais causas de morbimortalidade em crianças menores de 5 anos de idade, particularmente nos países em vias de desenvolvimento, responsáveis por mais de 4 milhões de óbitos por ano (WHO, 2013). Em Moçambique, no ano de 2010, este grupo de doenças foi a

RC: 84483

Disponível em: https://www.nucleodoconhecimento.com.br/saude/ambiente-domiciliar 
terceira causa de morbilidade e mortalidade entre as crianças menores de 5 anos de idade, tendo sido a pneumonia a mais grave em todas as regiões de Moçambique (UNICEF, 2014). Em 2011, as IRAs constituíram 52\% de atendimentos ambulatórios em pediatria (MISAU et al., 2013). Os principais fatores de risco ambientais para o desenvolvimento de doenças do trato respiratório foram indicados como, sazonalidade, poluição atmosférica, aglomeração, poluição doméstica e tabagismo (WHO, 1992b e VICTORIA, 1996).

Nas comunidades mais pobres, é frequente a queima de biomassa para cozinhar ou aquecer o ambiente, muitas vezes sem o devido cuidado com a emissão da fumaça e de outros resíduos provenientes da combustão levando a altas concentrações de poluentes nesses ambientes (WHO, 2012). Por outro lado, a fumaça do tabaco é o mais comum poluidor do ar domiciliar, e o grau de poluição depende do número de fumantes do domicílio e do número de cigarros fumados dentro de casa (CONSTANT et al., 2011; SPADA e LIPPI, 2014). Estes factos, resultam para as crianças, altas taxas de exposição ao fumo passivo (SPADA E LIPPI, 2014), podendo levar à resposta inflamatória da mucosa respiratória e ocorrência secundária, de doença respiratória, entre outras doenças, em crianças (CONSTANT et al., 2011; TOMOYUKI et al., 2014).

A presença de alérgenos no interior do domicílio, especialmente os pêlos de animais e ácaro do pó doméstico, tem sido relacionada à resposta inflamatória da mucosa respiratória e ao consequente estabelecimento de hiper-reatividade e ocorrência secundária de doença respiratória (PHELAN et al., 1994; RAMANI et al., 2016).

Ainda, outro elemento importante é a aglomeração de pessoas em domicílios, um fenómeno comum nas famílias de baixa renda devido, à elevada taxa de natalidade (GRANT, et al., 2012). Esse fator, associado à deficiência no acesso a água, saneamento e higiene, pode contribuir para aumento da incidência das doenças 
respiratórias (ARANHA et al., 2011), principalmente em crianças devido a sua susceptibilidade (STEWART et al., 2013).

Como a questão habitacional encontra-se intimamente ligada aos problemas de saúde, as abordagens e as intervenções no ambiente domiciliar tornaram-se um importante meio de combate as infecções respiratórias agudas, contribuindo, deste modo, para a melhoria do padrão de vida das comunidades (ARANHA et al., 2011). Este estudo teve por objetivo medir a proporção e identificar os principais fatores de risco do ambiente domiciliar associados à infecção respiratória aguda, na Localidade de Beleluane, Distrito de Boane, Província de Maputo, no sul de Moçambique.

\section{MÉTODOS}

Estudo transversal foi realizado em agregados familiares com crianças de 0 a 59 meses de idade, residentes na localidade de Beleluane, província de Maputo, no sul de Moçambique, entre os meses de Maio e Novembro de 2016. Os agregados familiares da localidade de Beleluane foram selecionados por amostragem aleatória por conglomerado (BUSSAB e MORETTIN, 2004), tendo como base os dados do cadastro da administração da localidade de Beleluane, antecedido de contatos com as autoridades do posto Administrativo de Matola-Rio e o chefe da localidade de Beleluane seguidos de reuniões realizadas com os chefes dos quarteirões das quatro (4) Células de Beleluane (A, B, C e D) onde foram explicados o âmbito e os objetivos do trabalho.

A localidade de Beleluane possuí um total de 2.156 famílias cadastradas e é constituída por 4 células. A cada célula corresponde um conjuntos de 300 a 350 agregados com crianças menores de 5 anos previamente demarcados pela administração da localidade de Beleluane. Os dados do posto administrativo permitiram estimar que haveria uma criança de até 5 anos a cada dois domicílios. Assim, para atingir o tamanho amostral desejado, seria necessário visitar 70

RC: 84483

Disponível em: https://www.nucleodoconhecimento.com.br/saude/ambiente-domiciliar 
agregados familiares na célula C e D e 95 agregados familiares na célula A e B. Para a amostragem dos agregados com crianças menores de cinco anos de idade, foram numeradas as quatro células e colocados em uma lista e foram sorteados aleatoriamente para visita. Em seguida, cada uma das esquinas dos quarteirões escolhidos foi numerada; uma dela foi sorteada e a partir desse ponto, sempre no sentido horário, foram visitados 70 e 95 domicílios consecutivos. Se não houvesse nesse agregado familiar criança menores de 5 anos, passava-se à quadra seguinte até atingir o número necessário de agregados familiares com criança menores de 5 anos. Foram no total visitados 330 agregados familiares.

Para recolha dos dados primeiro foram utilizados fontes primárias que ocorreu pelo meio de uma entrevista com os informantes chave (chefes ou representantes do agregado familiar), residente em Beleluane, que responderam um questionário estruturado, contendo perguntas específicas para estudar as variáveis propostas.

O questionário estava dividido em duas partes: (1) características sociais do agregado familiar, eram obtidas informações sobre características demográficas (sexo do chefe do agregado familiar, número de agregados familiares, e números de crianças menores de cinco anos), socioeconômicas da família (renda do agregado familiar e escolaridade dos pais), condições de habitação e saneamento básico (tipo de casa, tipo de revestimento do chão da casa, tipo de casa de banho, acesso a água, rede eléctrica, número de janelas, números de cómodos e aglomeração domiciliar) e ambientais (tipo de combustível para cozinhar, animais no domicílio e tabagismo domiciliar); (2) ocorrência de infecções respiratórias agudas nas crianças menores de 5 anos de idade no agregado familiar, foi definida pela presença de sinais e sintomas, foram referentes aos 14 dias anteriores à entrevista, para evitar possíveis vieses de memória. As enfermidades respiratórias foram referidas pelo chefe do agregado familiar, bem como as doenças mais frequentes em crianças menores de cinco anos. Para classificar os sintomas utilizou-se a Classificação Internacional de Doenças (WHO, 1992a).

RC: 84483

Disponível em: https://www.nucleodoconhecimento.com.br/saude/ambiente-domiciliar 
Em segundo lugar foram utilizadas fontes secundárias (prontuário médico) do Centro de Saúde de Beleluane para verificar as principais causas de busca de cuidados de saúde em Beleluane de Janeiro a Agosto. Nos prontuários foram recolhidas informações referentes a idade, sexo e residência da criança, o diagnóstico de infecções respiratórias agudas tais como quadro clínico no momento da consulta, data da consulta, e outras patologias não respiratórias.

A recolha de dados foi realizada pelo investigador principal e uma equipa de inquiridores (estudantes de graduação em Medicina da Universidade Eduardo Mondlane) previamente treinados para o trabalho de campo. Os dados recolhidos foram verificados diariamente para minimizar os erros de digitação. A verificação foi feita pelo supervisor do trabalho de campo.

Os dados foram digitados e analisados no programa SPSS versão 20. Em seguida, foi obtida uma listagem de frequências de todas as variáveis (desfecho, fatores de risco) e exame das suas distribuições. Quando possível, foram verificadas as medidas de tendência central (média, mediana e moda) e de dispersão (amplitude e desvio padrão). A seguir, sempre que necessário, foi feita uma recodificação com definição de pontos de corte para fatores de risco. A análise uni variada descritiva foi usada para caracterizar o agregado familiar conforme as variáveis selecionadas: características demográficas, características socioeconômicas, condições de habitação, acesso a água e saneamento e características ambientais domiciliares.

A proporção de crianças menores de 5 anos com infecção respiratória aguda na localidade de Beleluane foi obtida pelo seguinte cálculo: Número de crianças com IRA/Total de crianças. No que tange aos fatores relacionados com ambiente domiciliar, realizou-se análise multivariada, controlando-se simultaneamente diversos fatores de risco, através do método de regressão logística relacionando a variável dependente IRA nas duas semanas anterior ao inquérito (autorreferida), e as variáveis independentes socioeconómicos (escolaridade dos pais e renda do agregado familiar)

RC: 84483

Disponível em: https://www.nucleodoconhecimento.com.br/saude/ambiente-domiciliar 
e fatores ambientais domiciliares (aglomeração domiciliar, tipo de combustível para cozinhar, fumante no domicílio, animais no domicilio) a um intervalo de confiança de 95\% (IC95\%). Em seguida, foram analisados todos os fatores de risco com valor de $\mathrm{P}$ $<0,05$.

Atendendo as normas, o projeto foi autorizada pelo comité da ética e provincial de saúde de Maputo. Os chefes ou responsáveis do agregado familiar, foram informados do teor e objetivos da pesquisa e solicitado o consentimento, garantindo sigilo das informações colhidas.

\section{RESULTADOS}

Participaram do presente estudo 330 agregados familiares com crianças menores de 5 anos de idade da localidade de Beleluane. Do total de 330 famílias, 53.0\% eram chefiadas por mulheres, $66.4 \%$ eram constituídos por mais de cinco membros e $32.4 \%$ tinham duas ou mais crianças menores de 5 anos.

No que concerne às condições de habitação e saneamento básico, $56.7 \%$ dos agregados familiares residiam em casas de cimento, $34.8 \%$ em casas de caniço, e $8.5 \%$ em casas de chapas de zinco. De todas as casas visitadas, $40.9 \%$ tinham o chão revestido por terra, 54.2\% não tinham janelas e 61.2\% não possuíam corrente eléctrica. Nos agregados familiares foi observada a presença de quatro ou mais pessoas dividindo o quarto da criança (61.2\%), nessas famílias, $50.6 \%$ das crianças dormiam acompanhadas por 3 ou mais pessoas na cama. Adicionalmente foram observadas deficiências no acesso à água e saneamento, sendo que $77.3 \%$ dos agregados usavam água de fontes não melhoradas (tanques em camiões ou carregada em tambores) e 70.6\% usavam latrinas não melhoradas (construída com materiais precárias).

RC: 84483

Disponível em: https://www.nucleodoconhecimento.com.br/saude/ambiente-domiciliar 
No que concerne às condições socioeconómicos, 18.2\% dos homens (pais) e $36.1 \%$ das mulheres (mães) não sabiam ler nem escrever. De todas as casas visitadas, cerca de $50.9 \%$ tinham rendimento mensal abaixo de salário-mínimo $(\$ 58,00)$. ${ }^{6]}$

O ambiente domiciliar em Beleluane foi caracterizado pelo uso de combustíveis sólidos (lenha, carvão, resíduos da colheita agrícola) para cozinhar (66.1\%), e também foi observada a presença de animais domésticos (70.9\%) com maior presença de gatos $(50.9 \%)$. Adicionalmente foi observado que, entre os homens $34.8 \%$ era fumante, e fumavam em média $6.3 \pm 4.7$ cigarros, enquanto $12.4 \%$ das mulheres fumantes, fumavam em média $3.0 \pm 1.9$ cigarros, dentre os fumantes ativos $48.1 \%$ referiram fumar dentro das casas.

A infecção respiratória aguda em crianças menores de 5 anos foi referida em $41.5 \%$ dos agregados familiares (Figura 1). Para além das infecções respiratórias agudas foram ainda relatados outros problemas de saúde, sendo malária, diarreias e dermatites os mais comuns.

RC: 84483

Disponível em: https://www.nucleodoconhecimento.com.br/saude/ambiente-domiciliar 
Figura 1. Infecção respiratória aguda em crianças menores de 5 anos. *Malária, diarreias, dermatites

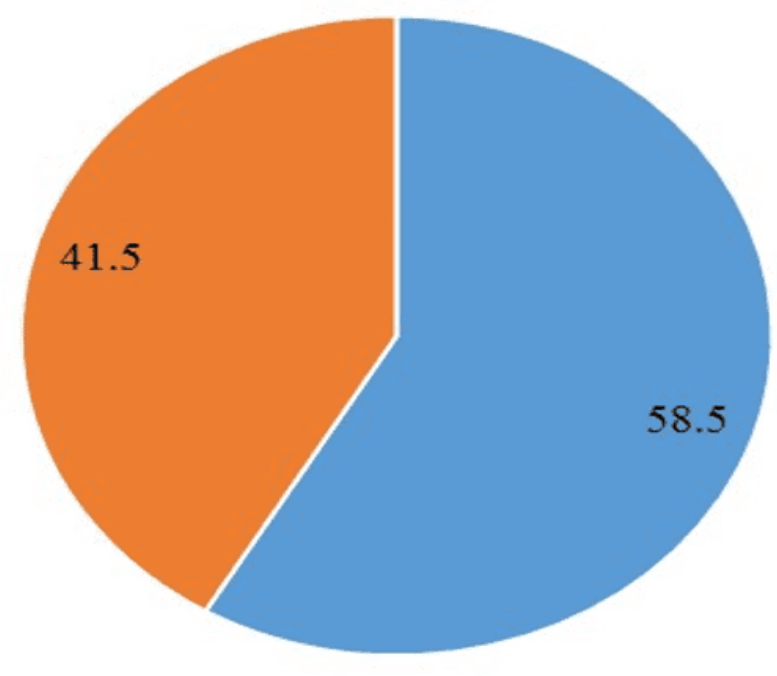

" Outros problemas de saúde* = Infecções respiratórias agudas

Fonte: Dados de pesquisa, 2016.

Os autorrelatos foram confirmados com os dados hospitalares sobre as principais razões de busca de cuidados de saúde para crianças menores de 5 anos na Localidade de Beleluane entre Janeiro e Agosto de 2016 (Figura 2). Verificou-se que, as infecções respiratórias agudas foram as principais causas da busca de cuidados de saúde em Beleluane de Janeiro a Agosto, onde registou-se a proporção em torno de 45.9 a $68.2 \%$ dos atendimentos pediátricos. Outros quadros clínicos incluíam diarreias, malária e infecções da pele.

RC: 84483

Disponível em: https://www.nucleodoconhecimento.com.br/saude/ambiente-domiciliar 
Figura 2. Infecções respiratórias agudas foram as principais causas da busca de cuidados de saúde em Beleluane de Janeiro a Agosto de 2016

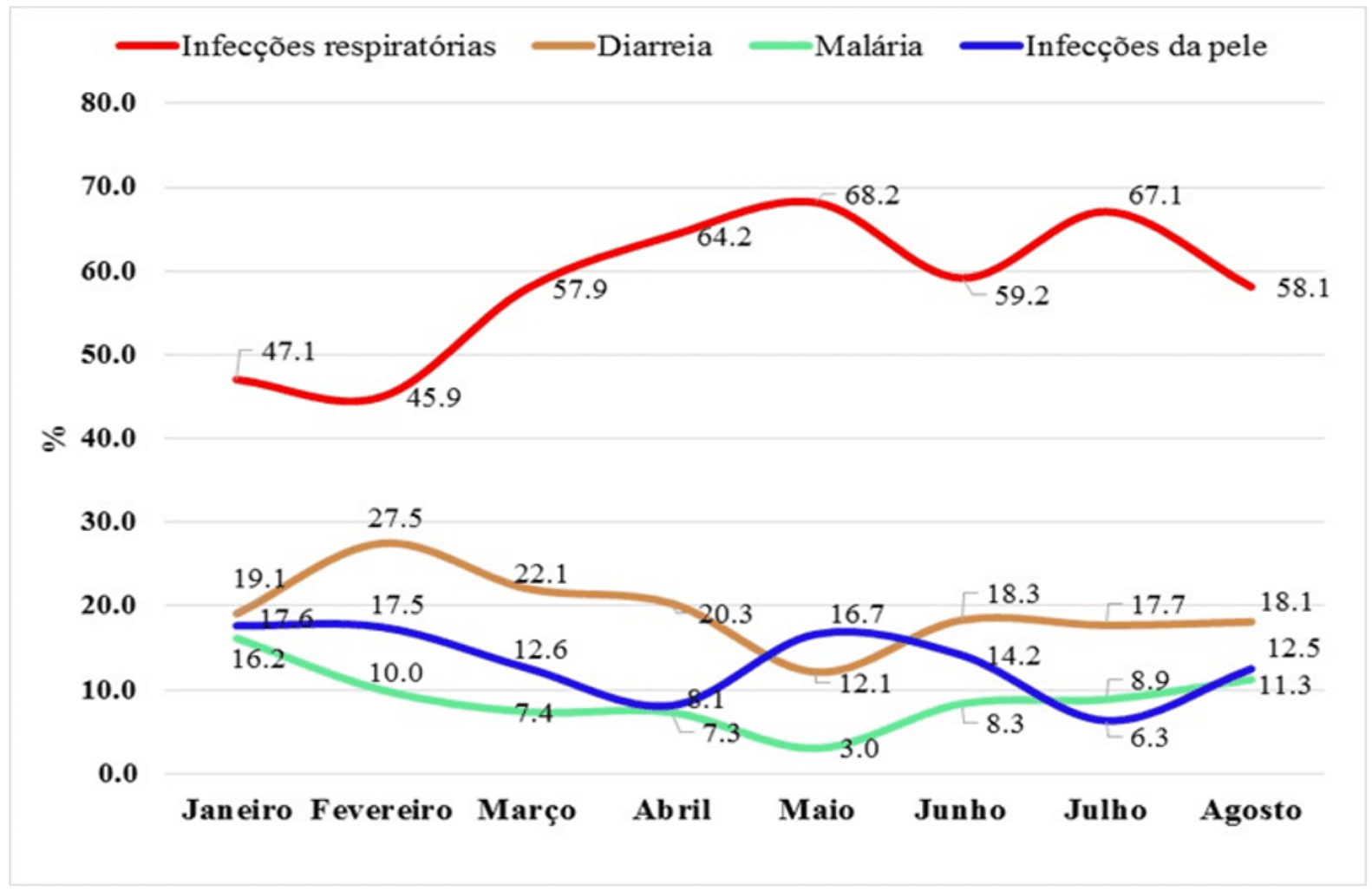

Fonte: Dados hospitalares, livro de registo do Centro de Saúde de Beleluane, Província de Maputo, sul de Moçambique).

Influência de fatores socioeconómicos (escolaridade dos pais e renda familiar) foi analisada (Tabela 1). Nos agregados familiares de Beleluane observou-se que a chance de morbidade por infecções respiratórias em crianças menores de cincos foi 10.265 vezes maior para crianças que tinham famílias com o rendimento mensal abaixo de salário-mínimo (\$58,00). E não houve associação estatisticamente significativa do desfecho com escolaridade dos pais.

Tabela 1. Determinantes socioeconómicos de infecções respiratórias agudas em Beleluane, província de Maputo, sul de Moçambique.

RC: 84483

Disponível em: https://www.nucleodoconhecimento.com.br/saude/ambiente-domiciliar 


\begin{tabular}{|c|c|c|c|}
\hline Fatores & OR & IC 95\% & $\mathbf{P}$ valor \\
\hline $\begin{array}{l}\text { Escolaridade do pai (Não saber ler nem } \\
\text { escrever) }\end{array}$ & 0.862 & $\begin{array}{l}0.395-1 . \\
883\end{array}$ & ${ }^{*} 0.711$ \\
\hline $\begin{array}{l}\text { Escolaridade da mãe (Não saber ler nem } \\
\text { escrever) }\end{array}$ & 0.687 & $\begin{array}{l}0.352-1 \\
340\end{array}$ & ${ }^{\star} 0.271$ \\
\hline Renda do agregado familiar $(\$ 58,00)$ & $\begin{array}{l}10 . \\
265\end{array}$ & $\begin{array}{l}5.168-20 \\
391\end{array}$ & $\begin{array}{ll}< & 0 . \\
001 & \end{array}$ \\
\hline
\end{tabular}

OR: Odds ratio; ${ }^{*} P>0.05$

A influência de fatores do ambiente domiciliares (tabagismo, uso de combustível sólido, presença de animais e aglomeração domiciliar) na ocorrência das infecções respiratórias agudas foi analisada (Tabela 2). Nos agregados familiares de Beleluane observou-se que a chance de morbidade por infecções respiratórias em crianças menores de 5 anos está associada com o tabagismo (um fumante ou mais no domicilio) (OR= 5.966;3.209-11.094), uso de combustível sólido para cozinhar (OR= 4.216; 2.02-8.802), presença de animais no interior do domicílio (OR=6.636; 3.111 14.155), e aglomeração domiciliar (quatro ou mais pessoas dividindo o quarto da criança) $(\mathrm{OR}=4.561 ; 2.493-8.345)$.

RC: 84483

Disponível em: https://www.nucleodoconhecimento.com.br/saude/ambiente-domiciliar 
Tabela 2. Determinantes do ambiente domiciliares de infecções respiratórias agudas em Beleluane, província de Maputo, sul de Moçambique.

\begin{tabular}{lccc}
\hline Factores & OR & IC 95\% & p valor \\
\hline Fumantes (Um fumante ou mais) & 5.966 & $3.209-11.094$ & $<0.001$ \\
Uso de combustíveis sólidos (Sim) & 4.216 & $2.020-8.802$ & $<0.001$ \\
Animais no interior do domicílio (Sim) & 6.636 & $3.111-14.155$ & $<0.001$ \\
Aglomeração pessoa/quarto ( $\geq 4$ pessoas) & 4.561 & $2.493-8.345$ & $<0.001$ \\
\hline OR: & Odds & & ratio
\end{tabular}

Fonte: Dados da pesquisa, 2016.

\section{DISCUSSÃO}

O contexto domiciliar (tabagismo, aglomeração, presença de animais, uso de combustíveis de sólidos) e socioeconômico (baixo rendimento) de Beleluane joga um papel relevante na ocorrência de infecções respiratórias agudas em crianças menores de 5 anos. Dados do presente estudo mostram altas taxas de IRAs referidas pelos chefes dos agregados familiares, e confirmadas no Centro de Saúde local como a principal razão de procura de cuidados de saúde quando comparada com outras doenças, como diarreias, malária e dermatites.

No presente estudo, revelou uma proporção mais elevada (41.5\%) de infecções respiratórias agudas em crianças menores de 5 anos dos agregados familiares de Beleluane, quando comparado a estudos realizados em diferentes contextos africanos nomeadamente Zimbabwe e na Tanzânia. Por exemplo, no estudo realizado no Zimbabwe a proporção de IRA autorreferida em crianças menores de cinco anos foi 16.0\% (MISHRA, 2003), na Tanzânia foi 31.1\% (BUKALASA, 2011). Um outro estudo similar realizado no Kenya mostrou uma proporção mais elevada em relação ao

RC: 84483

Disponível em: https://www.nucleodoconhecimento.com.br/saude/ambiente-domiciliar 
presente estudo, com a proporção de IRAs autorreferida, de 63.7\% (SIKPLIA et al., 2002).

Verificou-se que, as infecções respiratórias agudas foram as principais causas da busca de cuidados de saúde em Beleluane de Janeiro a Agosto, onde registou a proporção em torno de 45.9 a $68.2 \%$ dos atendimentos pediátricos. Além disto, este estudo revelou uma proporção mais elevada, quando comparado a outros estudos realizados no Brasil (MACEDO et al., 2003; DATASUS, 2011) mostraram proporções que variam entre $25.6 \%$ e $59.2 \%$ de atendimentos hospitalares pediátricos devido a IRAs. Por outro lado, dados do MISAU (2013) revelaram em 2011 que $52.0 \%$ dos atendimentos em Pediatria eram devido a IRAs, ressaltando a importância deste grupo de doenças na população moçambicana que tem vindo a crescer devido ao seu contributo nos atendimentos ambulatórios em diferentes unidades sanitárias de Moçambique e outros países com perfil socioeconômico similar, principalmente em comunidades pouco desenvolvidas.

No que se refere ao contexto socioeconômico, as crianças residentes em agregados familiares com renda baixa (definida neste estudo como sendo um rendimento mensal abaixo de salário-mínimo $(\$ 58,00)$ estão mais propensas as doenças respiratórias. Este cenário foi observado por diferentes pesquisadores que revelaram o aumento da predisposição para doenças respiratórias devido à baixa renda familiar, em Brasil (NASCIMENTO et al., 2004), Pelotas - Brasil (MACEDO et al., 2007), e Nepal (RANABHAT et al, 2015). Estes achados, podem ser explicados pelo facto das famílias desfavorecidas viverem sob condições precárias no que concerne ao acesso a bens, fontes de energia, tipo de habitação. Por outro lado, famílias carenciadas estão propensas a aglomeração domiciliar, e comportamentos que aumentam o risco das crianças desenvolverem quadros respiratórios (ARANHA et al., 2011 e GRANT et al., 2012). No caso de Beleluane por exemplo, observou-se que a maioria dos agregados familiares é chefiada por mulheres, com baixo nível de escolaridade, e baixa renda. 
Este perfil é consistente com as fracas condições de habitação, e consequente predisposição a fatores de risco para diferentes infecções.

A presença de um ou mais fumantes no domicílio foi uma variável importante na determinação das infecções respiratórias em crianças menores de 5 anos de Beleluane. Estes resultados estão em concordância com a maior parte dos estudos anteriores (GONÇALVES-SILVA et al., 2006; SEMPLE et al., 2011; SPADA e LIPPI, 2014) que confirmaram associações significativas entre a exposição domiciliar ao fumo de cigarro e o desenvolvimento de infecções respiratórias em crianças. Estes achados podem ser explicados pelo facto das crianças menores de 5 anos permanecerem mais tempo dentro de casa, e em contacto com os membros mais adultos, principalmente as mães, os pais e tios (SEMPLE et al., 2011). Desta forma a dose de exposição torna-se mais intensa a nível domiciliar, resultando em agravos da saúde (CONSTANT et al., 2011; SPADA e LIPPI, 2014).

Para o presente trabalho a aglomeração pessoa/quarto/cama apresentou uma associação com a ocorrência de infecções respiratórias em crianças menores de 5 anos de Beleluane. Em concordância com estudos de Prietsh et al., (2002) e Grant et al., (2012) também revelaram uma associação entre o rácio pessoa/quarto/cama e as infecções respiratórias. Sabe-se que a densidade de pessoas no mesmo quarto, associado as fracas condições de habitação, aumenta a chance de ocorrência de infecções cruzadas devido à maior oportunidade de contatos e transmissão de agentes patogénicos (ARANHA et al., 2011; GRANT et al., 2012). Em Beleluane, o grande número de membros dos agregados familiares pode resultar em alta densidade pessoa/quarto, principalmente em moradias com poucos cômodos. Esta característica, associada com as fracas condições de higiene devido a fraca circulação de ar (poucas ou nenhumas janelas), fraco acesso a água e saneamento constituem um importante contribuinte na disseminação das infecções respiratórias em Beleluane. 
Em Beleluane, outro fator que aumenta a chance de ocorrência das infecções respiratórias agudas é uso de combustíveis sólidos como fonte de energia para preparar os alimentos. Etiler et al., (2002) num estudo de coorte "(cohort)" prospectivo observaram na Turquia que, o uso de combustíveis sólidos aumentava o risco de ocorrência das infecções respiratórias agudas. Esta associação foi igualmente observada em Nepal por Ranabhat et al. (2015), e na Nigéria por Ezeh et al. (2014). Mais recentemente, Taksande e Yeole (2016), identificaram o uso de combustíveis sólidos entre os fatores de risco para infecções respiratórias agudas em crianças menores de 5 anos duma zona rural da Índia. Estes resultados podem ser explicados pelo facto das crianças nos seus primeiros anos de vida são geralmente carregadas pelas suas mães de costas ou permanece ao lado de suas mães ou parentes enquanto elas cozinham, sendo expostas aos diferentes poluentes de combustíveis sólidos. Por outro lado, as mães tendem a cozinhar no interior da casa nos dias frios, de maneiras a reaproveitar o fogo para o aquecimento da casa. Esta prática aumenta a circulação de poluentes da biomassa lenhosa no interior das casas (BATES et al., 2013; BUCHNER e REHFUESS, 2015).

Embora a teoria da higiene, postule que a exposição precoce aos animais domésticos seja um fator de proteção de sensibilização alérgica respiratória (JAAKKOLA 1991; FERREIRA, 2007), foi observado no presente estudo, que a presença de animais domésticos no interior do domicílio aumenta a chance de ocorrência de infecções respiratórias. Esta constatação é reforçada pela observação de Ramani et al., (2016), que defende que o contacto com os animais aumenta a sensibilidade alérgica e resulta na resposta inflamatória da mucosa respiratória e ocorrência secundária da asma e outras doenças respiratórias em crianças

\section{CONCLUSÃO}

Os resultados deste estudo mostram que a renda familiar joga um papel importante na ocorrência de infecções respiratórias em crianças menores de 5 anos. Os fatores

RC: 84483

Disponível em: https://www.nucleodoconhecimento.com.br/saude/ambiente-domiciliar 
ambientais domiciliares estão entre os principais determinantes das infecções respiratórias agudas nesse grupo etário na localidade de Beleluane, no sul de Moçambique.

O conhecimento detalhado dos fatores de risco modificáveis, observados nesse estudo, pode contribuir para minimizar as IRAs e suas complicações em crianças.

\section{REFERÊNCIAS}

ARANHA, Maria Aparecida et al. Relação da doença respiratória declarada pelos pais e factores socioeconómicos e culturais. Rev Paul Pediatr; v. 29, n.3, p.352-6, 2011.

BATES, Michael et al. Acute lower respiratory infection in childhood and household fuel use in Bhaktapur, Nepal. Environ Health Perspect, v.121, n. 5, p.637-42, 2013.

BUCHNER, Hannes; REHFUESS, Eva. Cooking and Season as Risk Factors for Acute Lower Respiratory Infections in African Children: A Cross- Sectional Multi-Country Analysis. PLoS ONE v.10, n.6, p.129-133, 2015.

BUKALASA, Joseph. Indoor Air Pollution, Social Inequality and Acute Respiratory Diseases in Children in Tanzania. Umea International School of Public Health, Umea University, Sweden, p. 44, 2011.

BUSSAB, Wilton; MORETTIN, Pedro. Estatística básica. 5a ed. São Paulo: Saraiva, P. 400, 2004.

CONSTANT, C. et al. Environmental tobacco smoke (ETS) exposure and respiratory morbidity in school age children. Rev Port Pneumol, v.17, n.1, p.20-6, 2011.

DATASUS-Brasília. Ministério da Saúde-Sistema de Informações Hospitalares do SUS 2011. Disponível em: http://www.datasus.gov.br/catalogo/sihsus.htm.Acesso em: 12 de Agosto de 2016.

RC: 84483

Disponível em: https://www.nucleodoconhecimento.com.br/saude/ambiente-domiciliar 
ETILER, Nilay; VELIPASAOGLU, Sevtap. AKTEIN, Mehmet. Incidence of acute respiratory infections and the relationship with some factors in infancy in Antalya, Turkey. Pediatric Int, n.44, p.64-9, 2002.

EZEH, Osita et al. The effect of solid fuel use on childhood mortality in Nigeria: evidence from the 2013 cross-sectional household survey. Environmental Health, p.13-113, 2014.

FERREIRA, Cristina; Seidman, Ernest. Alergia alimentar: actualização prática do pontos de vista gastrenterologias. J. Pediatric, n. 83, p.1-7, 2007.

GRANT, Cameron. et al. Risk factors for community-acquired pneumonia in preschool-aged children. Journal of Pediatrics and Child Health, v.48, n.5, p. 402-412, 2012.

GONÇALVES-SILVA, Regina et al. Household smoking and respiratory disease in under-five children. Cad. Saúde Pública, v. 22, n.3, p.579-586, 2006.

JAAKKOLA, Jouni et al. Low-level air polluition and upper respiratory infections in children. Am J public Health, n.8, p.1060-1063, 1991.

MINISTERIO DA SAÚDE (MISAU), Instituto Nacional de Estatística (INE) e ICF International (ICFI) Moçambique Inquérito Demográfico e de Saúde 2011. Calverton, Maryland, USA, 2013.

MISHRA, Vinod. Indoor air pollution from biomass combustion and acute respiratory illness in preschool age children in Zimbabwe. International Journal of Epidemiology, v.32, n.5, p.847-853, 2003.

NASCIMENTO, Luis et al. Análise hierarquizada dos factores de risco para pneumonia em crianças. Jornal Brasileiro de Pneumologia, v. 30, n.5, p.445 - 451, 2004. 
PHELAN, Peter, at al. Respiratory illness in children. 4th ed. Australia: Blackwell Scientific Publication; p. 27-51, 1994.

PRIETSCH, Silvio et al. Doença aguda das vias aéreas inferiores em menores de cinco anos: influência do ambiente doméstico e do tabagismo materno. J Pediatric, v.78, n.5, p.415-22, 2002.

RAMANI, Vinod et al. Acute Respiratory Infections among Under-Five Age Group Children at Urban Slums of Gulbarga city: A longitundinal Study. Int. j. Curr. Res. med. Sci, v.2 n.7, 2016.

RANABHAT, Chhabi et al. Consequence of indoor air pollution in rural area of Nepal: a simplified measurement approach. Frontiersin Public Health/ Environmental Health, v.5 n.3, 2015.

SELVARAJ, Kalaiselvi. et al. Acute respiratory infections among under-5 children in India: A situational analysis. Journal of Natural Science, Biology, and Medicine, v.5, n.1, p.15-20, 2014.

SEMPLE, Malcolm et al. Household tobacco smoke and admission weight predict severe bronchiolitis in infants independent of deprivation: prospective cohort study. PLoS One, v.6, n.7, p.22 - 425, 2011.

SIKOLIA, D et al. The prevalence of Acute Respiratory Infections And the associaded risk factors: A Study of children under five years of age in Kbera Lindi Village, Nairobi, Kenya. J. Natl. Inst. Public Health, v.5, n.1, 2002.

DE SOUSA, Clovis Arlindo et al. Respiratory diseases and associated factors: Population-based study in São Paulo, Brazil, 2008-2009. Rev Saúde Pública, v.46, n.1, p.16-25, 2012.

RC: 84483

Disponível em: https://www.nucleodoconhecimento.com.br/saude/ambiente-domiciliar 
SPADA, Ivete; LIPPI, Umberto. Tabagismo e sintomas respiratórios em crianças menores de cinco anos em determinada região da amazónia. Rev. Cient. Educ e Meio Ambiente, v.5, n.1, p.1-15, 2014.

STEWART, Jackon. et al. Risk factors for severe acute lower respiratory infections in young children. Croat Med J, n.54, p.110-21, 2013.

TAKSANDE, Amar; YEOLE, Mayuri. Risk factors of Acute Respiratory Infection (ARI) in under-fives in a rural hospital of Central India. Journal of Pediatric and Neonatal Individualized Medicine, v.5, n.1, 2016.

TOMOYUKI, Shibata et al. Childhood Acute Respiratory Infections and Household Environment in an Eastern Indonesian Urban Setting Int. J. Environ. Res. Public Health, n.11, p.12190-12203, 2014.

UNITED NATIONS CHILDREN'S FUND (UNICEF). Situação das Crianças em Moçambique. UNICEF. Moçambique, Maputo, 2014. Disponivel em(http://sitan.unicef.org.mz/). Acesso em: 25 de Setembro de 2016.

VICTORIA C. Factores de riesgo en las IRA bajas. In: Benguigui Y, Lópes-Antuñano FJ, Yunes J, editores. Infecciones respiratorias en niños. Washington: OPAS; p.4563, 1996.

WORLD HEALTH ORGANIZATION (WHO). ICD-10: international statistical classification of diseases and related health problems. WHO. 10 $0^{\text {th }}$, Geneva, 1992a.

WORLD HEALTH ORGANIZATION (WHO). Programme for the control of acute respiratory infections. Programme Report WHO/ARI/90.7.WHO, Geneva, 1992b.

WORLD HEALTH ORGANIZATION (WHO). "Country Profile of Environmental Burden of Disease". WHO, Geneva. 2009.

RC: 84483

Disponível em: https://www.nucleodoconhecimento.com.br/saude/ambiente-domiciliar 
WORLD HEALTH ORGANIZATION (WHO). Data base on Solid and Non Solid Fuel Usage. WHO, Geneva, 2012.

WORLD HEALTH ORGANIZATION (WHO). Pneumonia: Facts sheet; WHO Media Centre: Switzerland, Geneva, 2013.

\section{APÊNDICE - REFERÊNCIA DE NOTA DE RODAPÉ}

6. Salário-Mínimo de Moçambique: segundo artigo 85 da lei do trabalho (2016).

Enviado: Março, 2021.

Aprovado: Maio, 2021. 